\title{
Formulation of Presliding Domain Non-local Memory Hysteretic Loops Based Upon Modified Maxwell Slip Model
}

\author{
Krzysztof Jankowski $^{1} \cdot$ Michał Marszal $^{1} \cdot$ Andrzej Stefański $^{1}$
}

Received: 27 September 2016/Accepted: 20 March 2017/Published online: 28 March 2017

(c) The Author(s) 2017. This article is an open access publication

\begin{abstract}
In this paper, we investigate formulation of nonlocal memory effect hysteretic loops in presliding domain of friction by examining the dynamic responses of a singledegree-of-freedom friction oscillator. We modify the Maxwell slip model introducing characteristic features of asperities and perform detailed examination of the system from two perspectives. The first one, termed by us as $m i-$ croscale, presents contribution of each individual asperity to the total friction force, whereas the second one, termed by us as macroscale, concerns friction force behaviour of the whole model treated as the unity, with a special emphasis on non-local memory effect hysteretic loops formulation, complemented by time responses.
\end{abstract}

Keywords Dry friction $\cdot$ Non-local memory $\cdot$ Hysteretic effects $\cdot$ Maxwell slip model

\section{Introduction}

The common definition of friction says it is a reactive, resistive force between contacting interfaces in relative motion. Such a interpretation is descriptive, rather than strict, as this mechanical phenomenon reveals greatly nonlinear behaviour and incorporates range of phenomena to date not fully discovered. These phenomena, addressed in the literature, include: static friction, breakaway force and dynamic friction [1-3], presliding displacement [4], Stribeck effect [5-11], hysteretic effects [5, 6, 9, 12-29],

Krzysztof Jankowski

krzysztof.jankowski@p.lodz.pl

1 Division of Dynamics, Technical University of Łódź, Stefanowskiego 1/15, Łódź 90-924, Poland dwell-time and rising breakaway force [30-32], and stickslip motion [8, 12, 13, 15, 30-33]. Recently, the studies also extend to the analysis of synchronization of frictional systems, [34, 35]. It has commonly been assumed that due to vastness of friction scope it is challenging to include every frictional phenomena in a single model. However, newly appeared models try to include as many friction characteristics as possible, while providing accurate results. Most frequently, these characteristic features of friction allow to divide it into two subregions, the presliding regime and the pure sliding regime [4]. The first one refers to the microscopic displacement that takes place during the static phase, wherein the asperity forces are predominant and accordingly, the friction force is expressed as a function of displacement rather than velocity. Interesting part of aforementioned phenomena, occurring in both friction domains, stand hysteretic effects, which signify their presence in number of engineering systems like piezoelectric actuators [36, 37], structures being stressed beyond elastic limit, magnetic materials under altering electromagnetic field and here investigated friction force, as the result of microdisplacement [38-40].

The very definition of hysteretic effects depends on the system dynamic state and is formulated based on the division into two categories, [41]. The first one-hysteresis with local memory-reflects specific input-output relation, wherein impending system response depends upon its present output and future input. On the other hand, hysteresis with non-local memory extends it to incorporation of past extreme input values [42]. In case of friction, the division includes contact compliance, frictional memory and non-reversible friction characteristic. The first one originates from the spring-like behaviour of the friction force before the actual sliding, which emerges as the result of tangential stiffness of contacting surfaces. They give rise 
to microscopic displacement (also referred to as presliding or preliminary displacement) due to elastic deformation at the contact point or in its strict vicinity [4, 27, 43-45]. The second group obeys pure sliding domain, wherein system is subjected to oscillations. If these oscillations are considered as unidirectional, as propounded in [26], different paths followed by friction curve during acceleration and deceleration phases produce closed loop. The size of the loop increases for higher acceleration values [46], and friction force is lower for decreasing velocities than in case of increasing ones. Consequently, the dynamical behaviour is explained by the existence of frictional memory caused by a lag in the friction force. Further extension of this investigation to regressive oscillations (positive and negative amplitudes) results in non-reversible friction characteristic [47, 48], which can further account for presliding displacement.

The fundamentals and basic frictional behaviour, including hysteresis, are described by Al-Bender in [49]. He elaborates, step by step, aspects of friction and provides brief results of examination of generic friction model to obtain hysteresis behaviour for a given trajectory. In paper [50] he also outlines characteristic features of rate-independent hysteresis and presents response of a single-degree-of-freedom system consisting of mass attached to the hysteretic spring. Another interesting deliberation concerning hysteresis extended by elastic interactions of microasperities in dry friction is addressed in [51], wherein significance of asperity heights along with their elastic interactions is presented. It is shown that multistability of a single contact may lead to the hysteretic effects and discontinuity jumps and regarding the considered conditions, it is stated that velocity-independent dry friction is a direct consequence of that hysteresis. The paper [52] explains what is the area dependence and the load dependence of the coefficient of sliding friction in terms of hysteresis. Coefficients of hysteretic frictions are determined to eventually calculate generated stresses occurring in the examined rubber. Another author pointing out importance of understanding viscoelastic or hysteresis component of friction is Goda. In [53] he utilizes the concept of surface roughness to predict the viscoelastic friction and performs experimental investigation of the smooth surface to generate it, pointing out that hysteresis component of friction depends on both, macro- and microgeometry (surface roughness). Hysteretic friction with regard to surface topography is also addressed in [54], wherein investigation concerns hysteretic rubber friction, i.e. the ideally rigid, rough surface sliding upon a rubber plate. To do so, authors create 2D and $3 \mathrm{D}$ models to employ FE method for further analysis. In another work, [55], authors focus on tribological tests to determine hysteretic friction of rubber, whereas experimental and analytical study is presented in [56].
To date there has been extensive study devoted to hysteresis and hysteretic effects; however, in none of them hysteretic loops formulation mechanisms have been explained in details. To address this issue, in this paper, we undertake detailed investigation of the 5-asperity system, employing modified Maxwell slip model. We incorporate the forces acting on each particular asperity to study its frictional behaviour and contribution to overall friction force complemented by time responses. In the process, we introduce our micro- and macroscale definitions to diverge modelling perspectives and present step by step formulation of non-local memory hysteretic loops.

The paper is organized as follows: In Sect. 2 we introduce and extend the Maxwell slip model for the forthcoming analysis. We present hysteretic loops formulation from the viewpoint of micro- and macroscale in Sect. 3. Eventually, conclusions are drawn and presented in Sect. 4.

\section{Investigated Friction Model}

In this section we introduce the Maxwell slip model and implement a modification into it for the forthcoming analysis. Modification concerns characterization of asperities by their heights and normal and tangential stiffnesses. Additionally, normal separation distance between contacting surfaces is introduced. The general concept of this approach relies on $N$-elementary operators (asperities), connected in parallel, all subjected to the common input signal. The scheme of the Maxwell slip model, elaborated in [57-59], is presented in Fig. 1.

If the external signal $\delta_{t}$ is applied to the rigid flat, the operator exerts a tangential displacement, which is at the

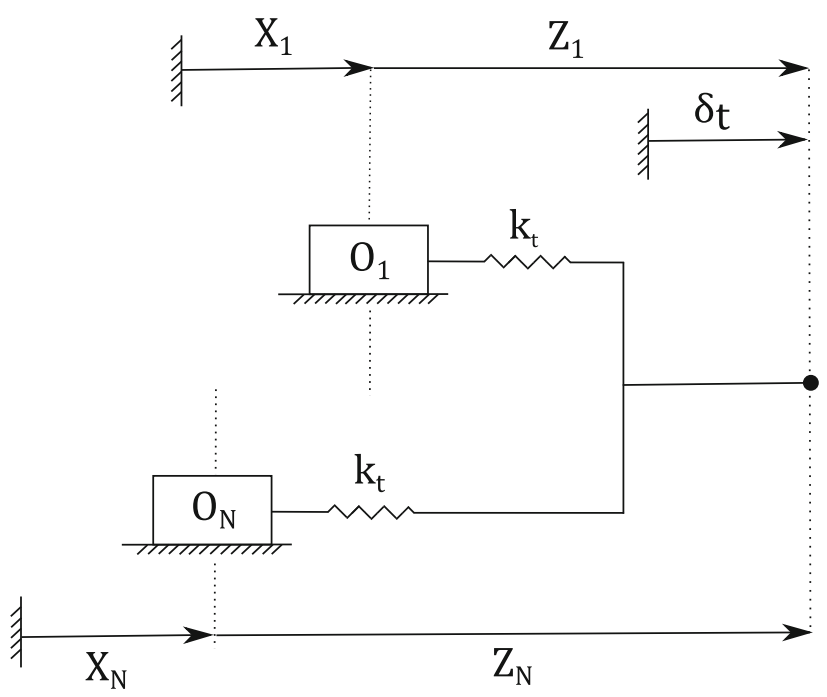

Fig. 1 Scheme of Maxwell slip model 
same time equal to the elongation of the tangent spring attached to the massless block $O_{i}$ from its equilibrium position, denoted as a state variable $Z_{i}$. Thus, the relative displacement of the given operator $X_{i}$ may be formulated as,

$X_{i}=\delta_{t}-Z_{i}$,

We modify the model by introducing normal springs between the operator and the surface. Such a formulation allows to derive both, normal and tangential forces, based on the physical properties of the asperities, i.e. their heights, normal and tangential stiffness and common separation distance, denoted by $h_{i}, k_{n}, k_{t}$ and $d$, respectively. Initially, the block $O_{i}$ sticks to the rigid flat and the tangential force, equal to the spring force $k_{t} Z_{i}$, increases with the increase of $\delta_{t}$ until the point where the spring force $k_{t} Z_{i}$ equals the static friction force between the contacting surfaces given by $\mu P_{i}=\mu k_{n}\left(h_{i}-d\right)$. Accordingly, we introduce the maximum allowable asperity deflection of $Z_{i}$, denoted by $\Delta_{i}$, as,

$\Delta_{i}=\frac{\mu k_{n}}{k_{t}}\left(h_{i}-d\right)$.

Please note that in the original Maxwell slip model, there are no mechanisms included to justify introduction of values of maximum allowable asperity deflection $\Delta_{i}$. Hence, the condition for sticking phase is given by,

$\left|Z_{i}\right|<\Delta_{i}$,

and the transition into pure sliding domain takes place if,

$\left|Z_{i}\right| \geq \Delta_{i}$.

One may notice that the relative displacement $X_{i}$ remains constant during sticking phase and the asperity deflection $Z_{i}$ remains constant during slipping phase. Accordingly, $X_{i}$ and $Z_{i}$ can be obtained from any of the following two sets of equations,

$\dot{X}_{i}= \begin{cases}0, & \text { stick regime } \\ \dot{\delta}_{t}, & \text { slip regime } .\end{cases}$

and

$\dot{Z}_{i}=\left\{\begin{array}{cl}\dot{\delta}_{t}, & \text { stick regime } \\ 0, & \text { stick regime },\end{array}\right.$

where $\dot{X}_{i}=\frac{\mathrm{d} X_{i}}{\mathrm{~d} t}, \dot{\delta}_{t}=\frac{\mathrm{d} \delta_{t}}{\mathrm{~d} t}$ and $\dot{Z}_{i}=\frac{\mathrm{d} Z_{i}}{\mathrm{~d} t}$ are the rate of change of $X_{i}, \delta_{t}$ and $Z_{i}$, respectively. Once the values of $Z_{i}$ are obtained by solving numerically any one of the two sets of equations given by Eqs. (5) and (6), the total friction force is determined by adding the elementary spring forces, taking the following form:

$$
F_{F}=\sum_{i=1}^{N} F_{\text {asp }(i)} \quad \text { where } \begin{cases}\mathrm{F}_{\text {asp (i) }}=\mathrm{k}_{\mathrm{t}} \mathrm{Z}_{\mathrm{i}}, & \text { for }\left|\mathrm{Z}_{\mathrm{i}}\right|<\Delta_{\mathrm{i}} \\ \mathrm{F}_{\text {asp }(\mathrm{i})}=\mathrm{k}_{\mathrm{t}} \Delta_{\mathrm{i}} \operatorname{sgn}\left(\mathrm{Z}_{\mathrm{i}}\right), & \text { for }\left|\mathrm{Z}_{\mathrm{i}}\right| \geq \Delta_{\mathrm{i}} .\end{cases}
$$

The non-local memory effect of friction can be obtained with this approach by choosing $\delta_{t}$ signal appropriately, as formulated in Eq. (8).

$$
\delta_{t}=A_{1} \sin (\omega t)+A_{2} \sin (2 \omega t) \text {, }
$$

where: $A_{1}, A_{2}$ are the corresponding signal amplitudes and $\omega$ is the excitation frequency.

Unless stated otherwise, we use parameter values listed in Table 1 to obtain the friction characteristics presented in the proceeding sections.

\section{Hysteretic Loops Formulation}

Al-Bender presented in [49] how the simple block system behaves under specific input signal applied to it. The signal was chosen to reveal the dependence between the friction force and the hysteretic displacement, with an inner loop placed inside the outer hysteresis loop. Delivered results of friction force vs displacement are rate independent, that is, the friction force vs position curve is not influenced by the speed of applied signal changes. In reference to Al-Benders works $[49,50]$ we decide to perform thorough investigation and describe mechanisms governing the formulation of non-local memory hysteretic loops, based on both, microand macroscale system analysis. By microscale, we understand approach, wherein contribution of each individual asperity to friction force is analysed, as opposed to macroscale, wherein the total friction force is derived and the whole model is treated as the unity. Therefore, blanket dynamical responses from macroscopic point of view are examined. As mentioned, order of asperity heights, i.e. $10^{-6} \mathrm{~m}$, is considered. For sake of consistency and comparison purposes, the exact conditions are maintained throughout the entire investigation-the exact set of asperities is put under investigation along with unaltered parameter values.

\subsection{Hysteretic Loops Formulation: Microscale}

A detailed investigation of friction forces, acting on individual asperities, modelled using the modified Maxwell slip model, elaborated in Sect. 2, is presented. To capture non-local memory hysteretic loops, as many as five asperities are used for the considered cases and the dynamic response of each particular asperity varies, depending on its height. Our examination can be extended further, since for our purposes we focus primarily on 
Table 1 Parameter values used in the numerical investigation

\begin{tabular}{llllll}
\hline$A_{1}(\mathrm{~m})$ & $A_{2}(\mathrm{~m})$ & $\omega(\mathrm{Hz})$ & $\mu(-)$ & $k_{n}(\mathrm{~N} / \mathrm{m})$ & $k_{t}(\mathrm{~N} / \mathrm{m})$ \\
$4 \times 10^{-4}$ & $4 \times 10^{-4}$ & 20 & 0.3 & $2.4 \times 10^{8}$ & $2.0 \times 10^{5}$ \\
\hline$d(\mathrm{~m})$ & $h_{1}(\mathrm{~m})$ & $h_{2}(\mathrm{~m})$ & $h_{3}(\mathrm{~m})$ & $h_{4}(\mathrm{~m})$ & $h_{5}(\mathrm{~m})$ \\
$1 \times 10^{-6}$ & $1.21667 \times 10^{-6}$ & $1.65000 \times 10^{-6}$ & $2.08330 \times 10^{-6}$ & $2.51670 \times 10^{-6}$ & $2.95000 \times 10^{-6}$ \\
\hline
\end{tabular}

asperity heights and separation distance parameters, keeping the normal and tangential stiffnesses constant, as listed in Table 1. Asperity heights are chosen to reflect every feasible asperity behaviour, while following the positive half of bell shape of the Gaussian distribution curve, since only asperities with their heights higher than the mean of asperity heights contribute into carry of the load. The whole system, and consequently each individual asperity, is subjected to the tangential kinematic excitation signal, given by a sum of two harmonic functions, according to Eq. (8). The system, initially at the steady-state condition, is subjected to excitation signal $\delta_{t}$ leading to the consecutive loading-unloading scenarios with a course shown in Fig. 2f. At first, a rapid growth of imposed excitation signal up to point $B$ takes place, which corresponds to the global maximum. Subsequently, signal velocity reversal occurs, signifying end of loading phase and followed by the unloading phase, indicated by segment $B-D$, wherein signal local minimum is attained at point $D$. Afterwards, displacement $\delta_{t}$ is increased below global maximum value denoting reloading scenario and in the proceeding step second unloading up to global minimum of imposed signal function at point $H$ is observed. Eventually, cycle ends with a loop closure at point $J$ and subsequently the whole cycle is repeated. It should be kept in mind that the path followed by the friction force is closed, under condition that the given asperity attains Coulomb force in the exact point for loading and second reloading, viz. reaching point $A$ on initial curve again. However, depending on asperity height, value of this point is different for every asperity (heightdependent). Clearly, the tallest asperity does not transition into pure sliding regime, since Coulomb force is not reached. Thus, loop closure for that asperity occurs at the uttermost point-global maximum of excitation signal (point $J$ ). Hence, to remain consistent and ensure closed hysteretic loop, in case of macroscale model, point $J$ is considered as the loop closure for all asperities.

Denoting asperities presented in Fig. 2a, b as Asperity 1, $2, \ldots, 5$, characterized by their heights, given, respectively, as $h_{1}, h_{2}, \ldots, h_{5}$, we analyse their individual responses.

Asperity 1. The increase in displacement signal $\delta_{t}$ is accompanied by the increase of friction force up to point $A$, where $f_{f}=1$. Consequently, as spring force threshold $\Delta$ is reached and friction force attains Coulomb friction force value, transition into pure sliding regime takes place. Sliding until point $B$ is observed, wherein velocity reversal of imposed excitation signal takes place, signifying beginning of unloading phase. Therefore, Asperity 1 sticks again and friction force increases in negative direction along with signal, until point $C$ is attained, wherein contact between asperity and the reference surface is broken yet again, starting another slipping phase. It is continued until signal velocity reversal occurs at point $D$. Since Asperity 1 is the shortest, small increase of excitation signal results in another sticking into sliding phase transition, as given by section $E-F$ and signified by friction force attaining Coulomb level. Similar behaviour is recorded for second unloading phase, wherein decrease of $\delta_{t}$ leads to decrease of $f_{f}$ values (sticking phase) and consequently, another regime switch at point $G$, ongoing to point $H$, occurs. Eventually, last scenario, i.e. second reloading is initiated, as indicated by increase of excitation signal. During sticking phase (section $H-I$ ) spring force is saturated once again, threshold value of $\Delta$ is reached at point $I$ and another sliding phase begins closing the whole cycle of Asperity 1. The friction force curve is closed at point $A$; however, due to the reasons mentioned above we assume it to be closed at point $J$.

Asperities 2 and 3 deliver qualitatively similar results. As previously, loading-unloading scheme is repeated and valid up to point $D$, where first reloading phase begins. Consequent increase of excitation signal $\delta_{t}$ is accompanied by increase of friction force (up to point $F$ ); however, in frames of presliding domain only, since these two asperities are that tall, that threshold value of $\Delta$ is not achieved for reloading case. As the result, transition into pure sliding regime does not take place. When velocity reversal of signal occurs, second unloading phase is initiated and friction force follows exactly the same sticking trajectory $F-D$, yet in the opposite direction. Since, unloading is continued, while friction force $f_{f}$ decreases to attain Coulomb friction value in negative direction, gross sliding is initiated at point $D$ once again, and continued until point $H$ is reached. Asperities 2 and 3 behave in the similar manner to Asperity 1 from that point.

Asperity characterized by height $h_{4}$ behaves distinctly, in comparison with the already considered cases. While subjected to loading scenario, it reveals the same patternincrease of displacement $\delta_{t}$ is followed by increase of friction force $f_{f}$ up to point $B$ (with presliding into gross sliding transition at point $A$ ). However, unloading the system, i.e. decreasing the displacement $\delta_{t}$ to the first local 
Fig. 2 Contribution of individual asperities to net friction force.

a $h_{1}=1.21667 \times 10^{-6} \mathrm{~m}$,

b $h_{2}=1.65000 \times 10^{-6} \mathrm{~m}$,

c $h_{3}=2.08330 \times 10^{-6} \mathrm{~m}$,

d $h_{4}=2.51670 \times 10^{-6} \mathrm{~m}$,

e $h_{5}=2.95000 \times 10^{-6} \mathrm{~m}$,

f excitation signal

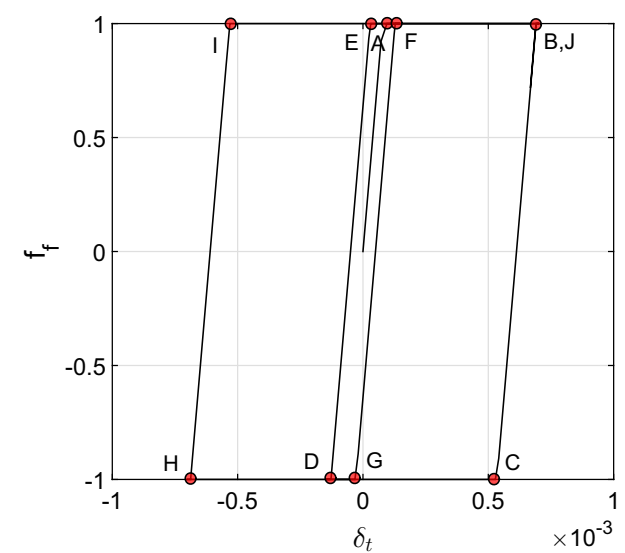

(a)

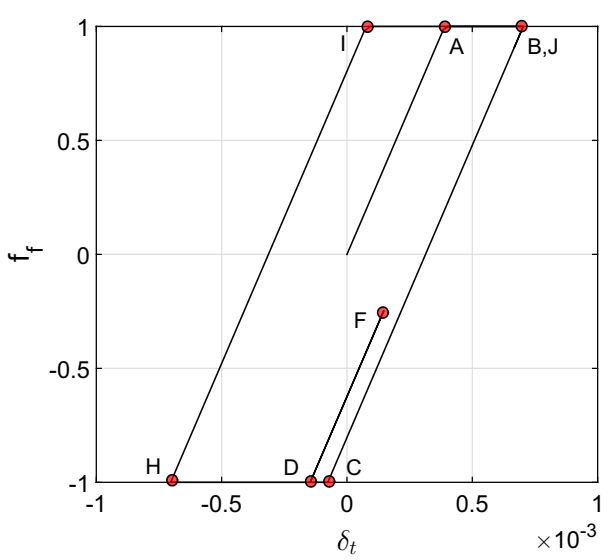

(c)

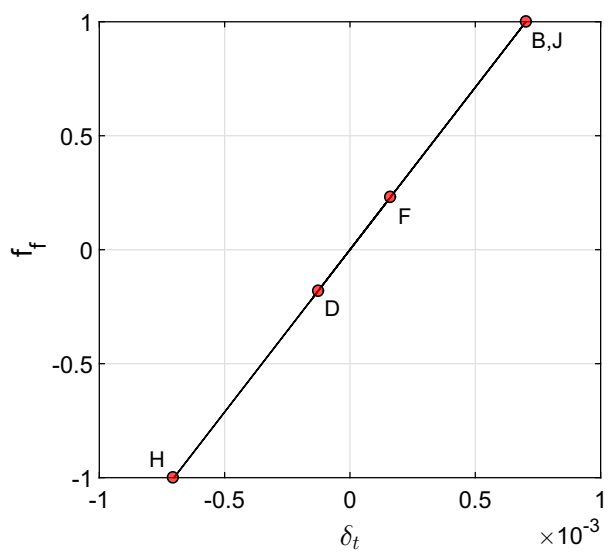

(e)

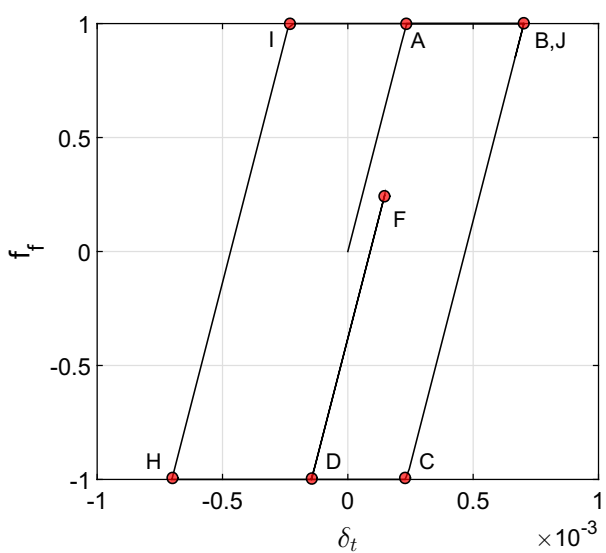

(b)

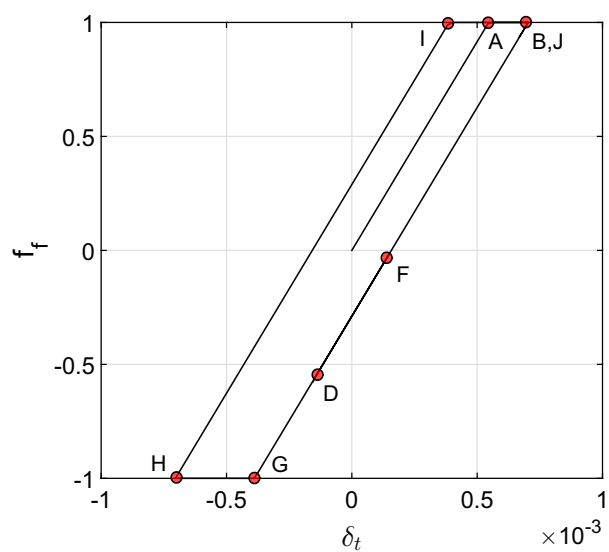

(d)

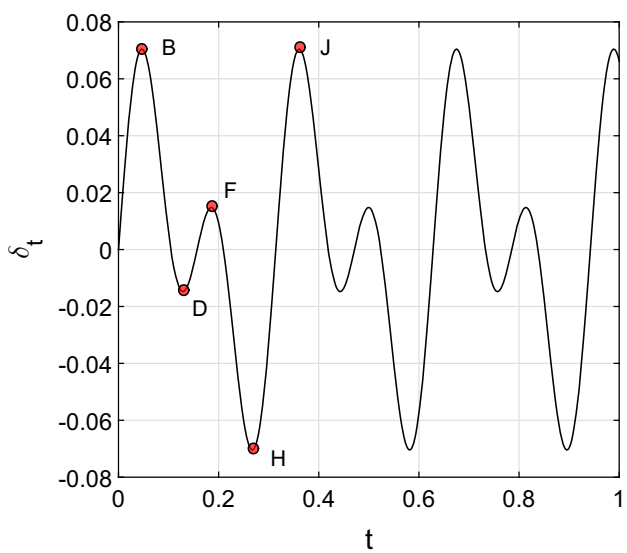

(f) minimum of excitation signal at point $D$, is not sufficient to reach spring force threshold value of $\Delta$ in negative direction, since Asperity 4 is too tall. Therefore, first reloading phase is considered as the reversal motion of first unloading phase. It follows the same path of sticking regime but in the opposite direction, as indicated by point $F$ lying at first unloading trajectory (section $D-F$ ). Subsequent decrease of signal results in second unloading phase, and this time provides negative displacement large enough to break junctions between contacting interfaces at point $G$. Consequently, Coulomb friction force is reached and gross sliding phase in negative direction is started, ongoing up to point $H$. Beginning at point $H$, the asperity follows the path $H-J$ in a similar manner to previous cases.

Last asperity of height $h_{5}$ reveals that neither scenario of loading-unloading-reloading delivers displacement large enough to force transition into gross sliding domain, since for this particular asperity height, boundary value of $\Delta$ is 
never reached. Consequently, any changes obey presliding regime only and friction force attains values below Coulomb friction level at any condition. Therefore, pictorially friction force vs imposed displacement relation is reproduced as the straight line, with all points $(A-J)$, corresponding to scenario change, being incorporated into that line, due to the boundary values of $\pm \Delta$ being not reached for any case. The uttermost points, i.e. global maxima (points $B, J$ ) and global minimum (point $H$ ), are also incorporated into that line.

It is observed from Fig. 2 that the friction force for each asperity increases or decreases linearly with the excitation signal in the sticking phase. The reason is that for the modified MS model, the rate of change of asperity deflection is given by $\dot{Z}_{i}=\dot{\delta}_{t}$ [Eq. (6)]. As discussed earlier, the sticking phase exists till the asperity deflection $\left|Z_{i}\right|<\Delta_{i}$, where $\Delta_{i}$ is the limiting asperity defection given

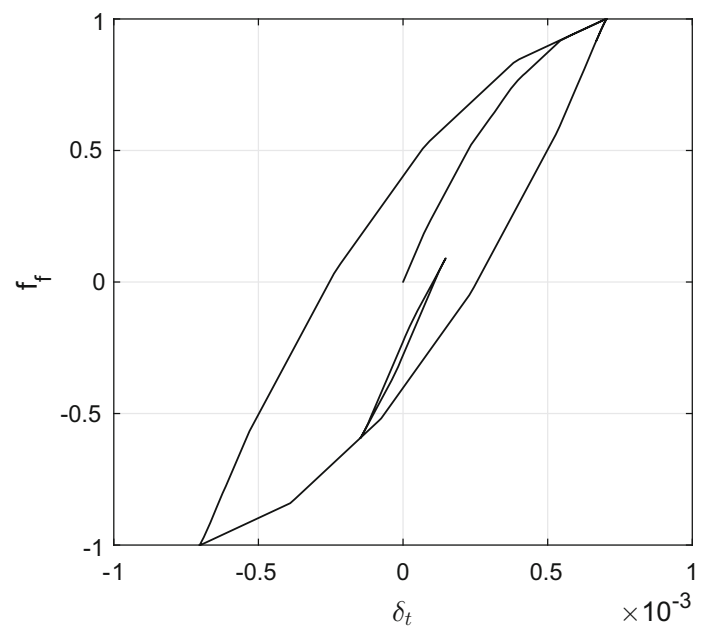

Fig. 3 Non-local memory hysteretic loop for the 5-asperity system by Eq. (2) for the modified MS model. The transition into pure sliding domain commences when $\left|Z_{i}\right| \geq \Delta_{i}$ and the friction force in this phase is given by the Coulomb friction force. It should be noted that the height of the fifth asperity $h_{5}=2.95000 \times 10^{-6} \mathrm{~m}$ is much larger compared to the heights of the other four asperities. Consequently, the fifth asperity never reaches the slipping phase. On the other hand, the transition of the shorter asperities into the pure sliding domain is faster than their higher counterparts and also, the sliding continues for longer time period. This results in larger hysteretic loop for the shorter asperities.

\subsection{Hysteretic Loops Formulation: Macroscale}

If the summation of the responses of investigated asperities is performed, the total friction force characteristic in relation to imposed tangential displacement can be obtained, which is the subject of this section. Macroscopic response of the system whole model treated as the unity, thus termed by us as macroscale, is considered. The exact set of 5 asperities, with heights listed in Table 1, is investigated. The external excitation signal $\delta_{t}$ remains unchanged, as well. Figure 3 depicts total friction force, derived from summation of contributions of individual asperities, analysed in previous section. As presented, as many as five asperities are sufficient to capture evident non-local memory hysteretic loop. The whole system remains sticking, since not every asperity junction has been broken, even though some of the asperities already slip, as discussed earlier and presented in Fig. 2.

Succeeding macroscale analysis is performed simultaneously based on results shown in Figs. 4 and 5, with the exact loading scenarios: loading curve $(0,0)-B$, unloading curve $B-D$, reloading curve $D-F$, second unloading curve
Fig. 4 Step by step formulation of non-local memory hysteretic loop, $X$-average asperity deflection, $\delta_{t}$-excitation signal, $f_{f}$-total friction force exerted by the system.

Corresponding segments refer to system behaviour indicated by Fig. 5
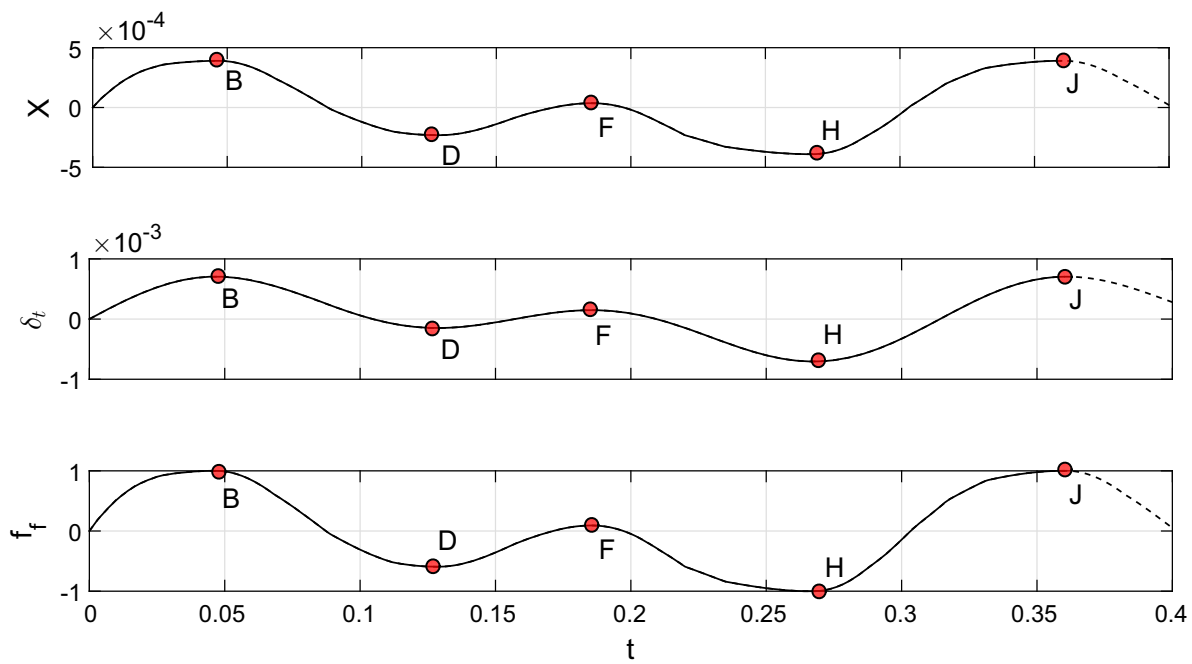
$F-H$, second reloading curve $H-J$. Corresponding course of imposed excitation signal is depicted in Fig. $2 \mathrm{f}$.

In the first step (Fig. 4), for increasing values of imposed excitation signal $\delta_{t}$, corresponding to loading scenario, increase of average asperity displacement $X$ is observed and consequently friction force is raised, Fig. 5a. Friction force asymptotically reaches its maximum value, since the imposed excitation signal is further increased to its global maximum (point $B$ ). Even though Coulomb friction force has been attained by some of the asperities (Fig. 2) and thus they begin to slip, the whole system does not transition into pure sliding domain, as not every asperity junction has been broken (Fig. 2e). Total friction force value is not kept constant and do not reach Coulomb friction force. Afterwards, the imposed signal velocity reversal takes place at point $B$, signifying the unloading scenario, as depicted in Fig. $5 \mathrm{~b}$ and given by the curve $B-D$ in Fig. 4. Rapid decrease of friction force is denoted,
Fig. 5 Step by step formulation of non-local memory hysteretic loop. a initial loading, b unloading, c reloadingbeginning of hysteretic loop formulation, $\mathbf{d}$ second unloading-closure of hysteretic loop, e second reloading — end of cycle, f excitation signal

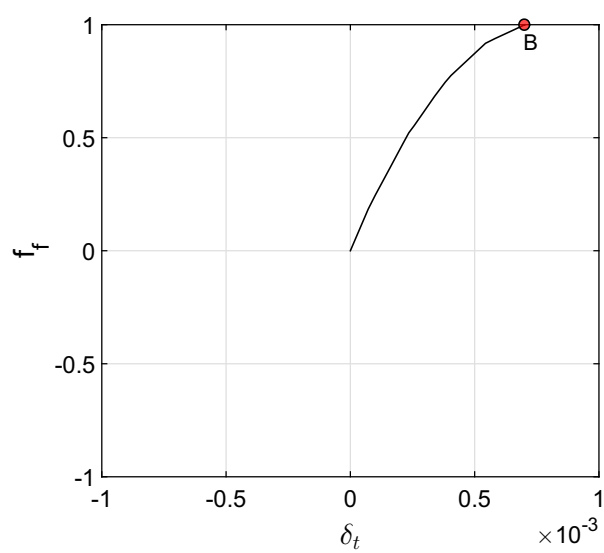

(a)

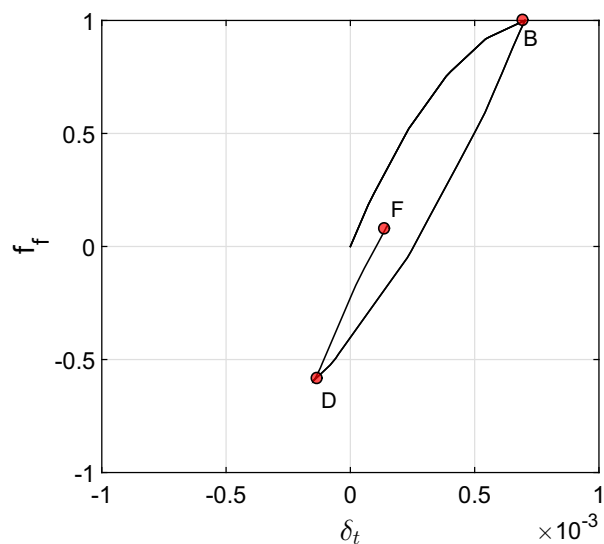

(c)

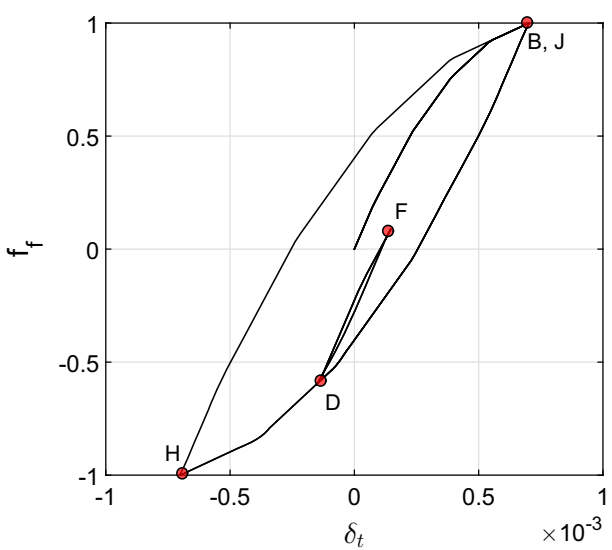

(e)

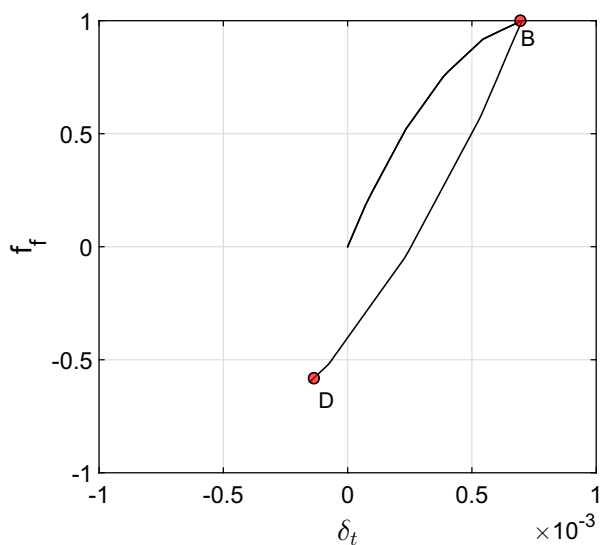

(b)

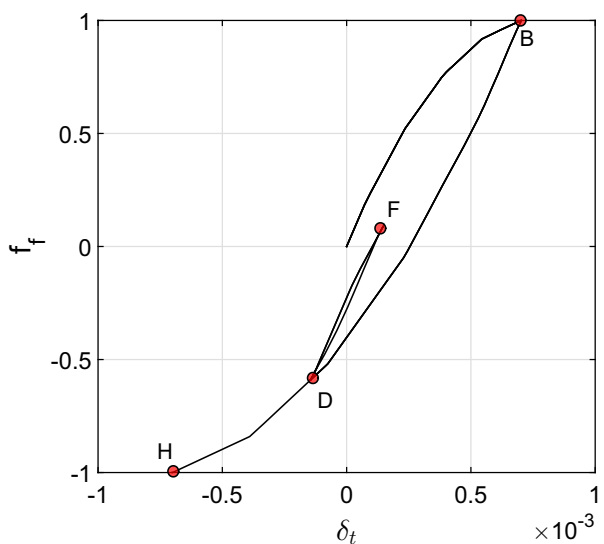

(d)

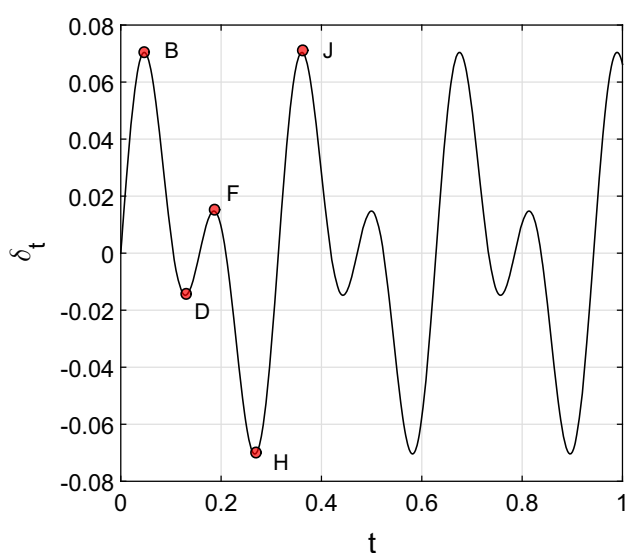

(f) 
as both excitation signal $\delta_{t}$ and thus average asperity displacement $X$ decreases. Again, some of the asperities already slip while unloading, however, not each of them (Fig. 2). This situation remains valid, until another imposed signal velocity reversal takes place, signifying first reloading phase, beginning at point $D$, Fig. 5c. Consequently, as excitation signal begins to increase, increase of average asperity deflection is observed and corresponding to its rise of friction force. It should be noted that according to imposed signal course (Fig. 2f), values it attains are significantly lower than for the initial loading curve and this applies to resultant friction force values as well. Therefore, as the consequence of the succeeding second unloading scenario (curve $F-D$ ) non-local memory effect hysteretic loop is formulated, Fig. 5d. That is signified by the exact values of friction force attained for both cases-during loading and unloading, pictorially justified as the friction curve returning to the initial course at the exact point, where the hysteretic loop emerged (point $D$ ). Such a trajectory proves non-local memory effect, while regardless of velocity reversals, friction force vs imposed excitation signal recovered at the very same point of the initial curve. The second unloading scenario continues, until maximum deflection in negative direction is reached, as shown by point $H$ in Fig. 5e. Consecutive second reloading scenario is indicated by, yet another increase of imposed excitation signal $\delta_{t}$ and corresponding to it increase in value of average asperity displacement $X$ and friction force (Fig. 5f). It leads to the local hysteretic loop closure, assumed to take place at point $J$.

Figure 5 depicts step by step formulation of the nonlocal memory hysteretic loop. Taking into consideration excitation signal reproducing function of several maxima and minima, non-local memory effect emerges as the consequence of past extrema and is visualized as the closed loop occurring inside local hysteretic loop. It should be noted that altering the amplitude values of harmonic functions included in Eq. (8) results in different sizes of hysteretic loop.

\section{Conclusions}

We described mechanisms governing the formulation of non-local memory hysteretic loops, based on both, microand macroscale analysis of the modified Maxwell slip model subjected to single-degree-of-freedom oscillator system. System consisting of five asperities was introduced, wherein every feasible asperity behaviour was considered. At first, in frames of microscale, the contribution of particular asperities to the total friction force along with thorough description of frictional dynamics was elaborated. Afterwards, it was complemented by macroscale responses of the whole model treated as the unity and the step by step formulation of non-local hysteretic loop was demonstrated along with simultaneous study of time responses. We investigated influence of asperity height and normal separation distance on regime transition and proved once again, that the whole system does not transition into gross sliding domain, unless all asperity junctions have been broken. We showed that system is rate independent and explained what the consequences of excitation signal alteration are.

Acknowledgements This work has been supported by Polish National Centre of Science (NCN) under Project No. DEC-2012/06/ A/ST8/00356.

Open Access This article is distributed under the terms of the Creative Commons Attribution 4.0 International License (http://creative commons.org/licenses/by/4.0/), which permits unrestricted use, distribution, and reproduction in any medium, provided you give appropriate credit to the original author(s) and the source, provide a link to the Creative Commons license, and indicate if changes were made.

\section{References}

1. Rabinowicz, E.: The nature of the static and kinetic coefficients of friction. J. Appl. Phys. 22(11), 1373-1379 (1951)

2. Johannes, V., Green, M., Brockley, C.: The role of the rate of application of the tangential force in determining the static friction coefficient. Wear 24(3), 381-385 (1973)

3. Richardson, R., Nolle, H.: Surface friction under time-dependent loads. Wear 37(1), 87-101 (1976)

4. Courtney-Pratt, J., Eisner, E.: The effect of a tangential force on the contact of metallic bodies. Proc. R. Soc. Lond. A 238, 529-550 (1957)

5. Lampaert, V., Al-Bender, F., Swevers, J.: Experimental characterization of dry friction at low velocities on a developed tribometer setup for macroscopic measurements. Tribol. Lett. 16(1-2), 95-105 (2004)

6. Janssens, T., Al-Bender, F., Van Brussel, H.: Experimental characterisation of dry and lubricated friction on a newly developed rotational tribometer for macroscopic measurments. In: Proceedings of the International Conference on Noise and Vibration Engineering, pp. 857-869 (2008)

7. Kröger, M., Neubauer, M., Popp, K.: Experimental investigation on the avoidance of self-excited vibrations. Philos. Trans. R. Soc. A 366(1866), 785-810 (2008)

8. Bristow, J.: Kinetic boundary friction. Proc. R. Soc. Lond. A 189, 88-102 (1947)

9. Yanada, H., Sekikawa, Y.: Modeling of dynamic behaviors of friction. Mechatronics 18(7), 330-339 (2008)

10. Armstrong-Hélouvry, B., Dupont, P., De Wit, C.C.: A survey of models, analysis tools and compensation methods for the control of machines with friction. Automatica 30(7), 1083-1138 (1994)

11. Jacobson, B.: The stribeck memorial lecture. Tribol. Int. 36(11), 781-789 (2003)

12. Sampson, J., Morgan, F., Reed, D., Muskat, M.: Studies in lubrication: Xii. Friction behavior during the slip portion of the stick-slip process. J. Appl. Phys. 14(12), 689-700 (1943)

13. Bell, R., Burdekin, M.: A study of the stick-slip motion of machine tool feed drives. Proc. Inst. Mech. Eng. 184(1), 543-560 (1969) 
14. Ko, P.L., Brockley, C.: The measurement of friction and frictioninduced vibration. J. Lubr. Technol. 92(4), 543-549 (1970)

15. Antoniou, S., Cameron, A., Gentle, C.: The friction-speed relation from stick-slip data. Wear 36(2), 235-254 (1976)

16. Sakamoto, T.: Normal displacement and dynamic friction characteristics in a stick-slip process. Tribol. Int. 20(1), 25-31 (1987)

17. Lee, R.T., Yang, C.R., Chiou, Y.C.: A procedure for evaluating the positioning accuracy of reciprocating friction drive systems. Tribol. Int. 29(5), 395-404 (1996)

18. Marui, E., Endo, H., Hashimoto, M., Kato, S.: Some considerations of slideway friction characteristics by observing stick-slip vibration. Tribol. Int. 29(3), 251-262 (1996)

19. Yang, C.R., Lee, R.T., Chiou, Y.C.: Study on dynamic friction characteristics in reciprocating friction drive system. Tribol. Int. 30(10), 719-731 (1997)

20. Van De Velde, F., De Baets, P.: The relation between friction force and relative speed during the slip-phase of a stick-slip cycle. Wear 219(2), 220-226 (1998)

21. Yang, C.R., Chiou, Y.C., Lee, R.T.: Tribological behavior of reciprocating friction drive system under lubricated contact. Tribol. Int. 32(8), 443-453 (1999)

22. Liang, J.W., Feeny, B.F.: Identifying Coulomb and viscous friction in forced dual-damped oscillators. J. Vib. Acoust. 126(1), 118-125 (2004)

23. Nouri, B.M.: Friction identification in mechatronic systems. ISA Trans. 43(2), 205-216 (2004)

24. Stefański, A., Wojewoda, J., Wiercigroch, M., Kapitaniak, T.: Regular and chaotic oscillations of friction force. Proc. Inst. Mech. Eng. C J. Mech. Eng. Sci. 220(3), 273-284 (2006)

25. Wojewoda, J., Stefański, A., Wiercigroch, M., Kapitaniak, T.: Hysteretic effects of dry friction: modelling and experimental studies. Philos. Trans. R. Soc. A 366(1866), 747-765 (2008)

26. Hess, D., Soom, A.: Friction at a lubricated line contact operating at oscillating sliding velocities. J. Tribol. 112(1), 147-152 (1990)

27. Liang, J.W., Feeny, B.: Dynamical friction behavior in a forced oscillator with a compliant contact. J. Appl. Mech. 65(1), 250-257 (1998)

28. Ibrahim, R., Zielke, S., Popp, K.: Characterization of interfacial forces in metal-to-metal contact under harmonic excitation. J. Sound Vib. 220(2), 365-377 (1999)

29. Guo, K., Zhang, X., Li, H., Meng, G.: Non-reversible friction modeling and identification. Arch. Appl. Mech. 78(10), 795-809 (2008)

30. Rabinowicz, E.: The intrinsic variables affecting the stick-slip process. Proc. Phys. Soc. 71(4), 668 (1958)

31. Kato, S., Matsubayashi, T.: On the dynamic behavior of machine tool slideway: 1st report, characteristics of static friction in "stick slip" motion. Bull. JSME 13(55), 170-179 (1970)

32. Kato, S., Sato, N., Matsubayashi, T.: Some considerations on characteristics of static friction of machine tool slideway. J. Lubr. Technol. 94(3), 234-247 (1972)

33. Bo, L.C., Pavelescu, D.: The friction-speed relation and its influence on the critical velocity of stick-slip motion. Wear 82(3), 277-289 (1982)

34. Marszal, M., Saha, A., Jankowski, K., Stefański, A.: Synchronization in arrays of coupled self-induced friction oscillators. Eur. Phys. J. Spec. Top 225(13-14), 2669-2678 (2016)

35. Marszal, M., Stefański, A.: Parameter study of global and cluster synchronization in array of dry friction oscillators. Phys. Lett. A 381(15), 1286-1301 (2017)

36. Ge, P., Jouaneh, M.: Modeling hysteresis in piezoactuators. Precis. Eng. 17(3), 211-221 (1995)

37. Goldfarb, M., Celanovic, N.: A lumped parameter electromechanical model for describing the nonlinear behavior of piezoelectric actuators. J. Dyn. Syst. Meas. Control 119(3), 478-485 (1997)
38. Futami, S., Furutani, A., Yoshida, S.: Nanometer positioning and its micro-dynamics. Nanotechnology 1(1), 31 (1990)

39. Swevers, J., Al-Bender, F., Ganseman, C.G., Projogo, T.: An integrated friction model structure with improved presliding behavior for accurate friction compensation. IEEE Trans. Autom. Contr. 45(4), 675-686 (2000)

40. Kuhnen, K., Janocha, H.: Adaptive inverse control of piezoelectric actuators with hysteresis operators. In: Control Conference (ECC), 1999 European, IEEE, pp. 791-796 (1999)

41. Mayergoyz, I.: Mathematical Models of Hysteresis. Springer, Berlin (1991)

42. Berényi, P., Horváth, G., Lampaert, V., Swevers, J.: Nonlocal hysteresis function identification and compensation with neural networks. IEEE Trans. Instrum. Meas. 54(6), 2227-2238 (2005)

43. Harnoy, A., Friedland, B., Rachoor, H.: Modeling and simulation of elastic and friction forces in lubricated bearings for precise motion control. Wear 172(2), 155-165 (1994)

44. Liang, J., Feeny, B.: Identifying Coulomb and viscous friction from free-vibration decrements. Nonlinear Dyn. 16(4), 337-347 (1998)

45. Liang, J., Feeny, B.: A comparison between direct and indirect friction measurements in a forced oscillator. J. Appl. Mech. 65, 783-785 (1998)

46. Olsson, H., Åström, K.J., De Wit, C.C., Gäfvert, M., Lischinsky, P.: Friction models and friction compensation. Eur. J. Control 4(3), 176-195 (1998)

47. Powell, J., Wiercigroch, M.: Influence of non-reversible Coulomb characteristics on the response of a harmonically excited linear oscillator. Mach. Vib. 1(2), 94-104 (1992)

48. Wiercigroch, M.: Comments on the study of a harmonically excited linear oscillator with a Coulomb damper. J. Sound Vib. 167(3), 560-563 (1993)

49. Al-Bender, F.: Fundamentals of friction modeling. In: Proceedings, ASPE Spring Topical Meeting on Control of Precision Systems, MIT, April 11-13, 2010, ASPE-The American Society of Precision Engineering, pp. 117-122 (2010)

50. Al-Bender, F., Symens, W., Swevers, J., Van Brussel, H.: Theoretical analysis of the dynamic behavior of hysteresis elements in mechanical systems. Int. J. Non-Linear Mech. 39(10), 1721-1735 (2004)

51. Caroli, C., Nozieres, P.: Hysteresis and elastic interactions of microasperities in dry friction. Eur. Phys. J. B 4(2), 233-246 (1998)

52. Yandell, W.: A new theory of hysteretic sliding friction. Wear 17(4), 229-244 (1971)

53. Goda, T.: On the viscoelastic component of rubber friction. In: VI. InterNatl. Engineering Symposium at Banki, Budapest, 17 Nov 2016, InterNatl. Engineering Symposium, paper 20 (2016)

54. Pálfi, L., Békési, N., Goda, T., Váradi, K., Czifra, Á.: Fe simulation of the hysteretic friction considering the surface topography. Period. Polytech. Mech. Eng. 52(2), 83 (2008)

55. Pálfi, L., Váradi, K.: Hysteretic friction of rubber in tribological tests. Period. Polytech. Mech. Eng. 54(2), 63 (2010)

56. Lindner, M., Kröger, M., Popp, K., Blume, H.: Experimental and analytical investigation of rubber friction. Safety 200, 300 (2004)

57. Rizos, D.D., Fassois, S.D.: Presliding friction identification based upon the maxwell slip model structure. Chaos 14(2), 431-445 (2004)

58. Ruderman, M., Bertram, T.: Modified maxwell-slip model of presliding friction. In: Proceedings of 18th IFAC World Congress, pp. 10764-10769 (2011)

59. Vo-Minh, T., Tjahjowidodo, T., Ramon, H., Van Brussel, H.: A new approach to modeling hysteresis in a pneumatic artificial muscle using the maxwell-slip model. IEEE/ASME Trans. Mechatron. 16(1), 177-186 (2011) 